\title{
A Generative Lexicon Perspective for Adjectival Modification
}

\author{
Patrick Saint-Dizier \\ IRIT-CNRS, Université Paul sabatier \\ 118 route de Narbonne F-31062 Toulouse Cedex France \\ stdizier@irit.fr
}

\begin{abstract}
This paper presents a semantic interpretation of adjectival modification in terms of the Generative Lexicon. It highlights the elements which can be borrowed from the GL and develops limitations and extensions. We show how elements of the Qualia structure can be incorporated into semantic composition rules to make explicit the semantics of the combination adjective + noun.
\end{abstract}

\section{Aims}

Investigations within the generative perspective aim at modelling, by means of a small number of rules, principles and constraints, linguistic phenomena at a high level of abstraction, level which seems to be appropriate for research on multi-linguism and language learning.

Among works within the generative perspective, one of the most innovative is the Generative Lexicon (GL) (Pustejovsky 91, 95) which introduces an abstract model opposed to sense enumeration lexicons. The GL is based (1) on the close cooperation of three lexical semantic structures: the argument structure, the aspectual structure and the Qualia structure (with four roles: Telic, Agentive, Constitutive and Formal), (2) on a detailed type theory and a type coercion inference rule and (3) on a refined theory of compositionality. The Generative Lexicon investigates the problem of polysemy and of the multiplicity of usages from a core sense of a lexeme and shows how these usages can be analyzed in terms of possible type shiftings w.r.t. the type expected by the core usage. Type shifting is modelled by a specific inference mechanism: type coercion.

In this paper, the following points are addressed:

- Generative systems require a clear analysis of the notions of word-sense and of sense delimitation. Depending on the strategy adopted (e.g. large number of narrow senses for a lexeme as in WordNet, or very few but large senses as in many Al works), the nature and the scope of generative operations may be very different.

- The Qualia structure is a complex structure, quite difficult to describe, in spite of evidence of its existence, in particular for the Telic role, (explored e.g. in the EuroWordNet project, the European WordNet). Qualias are well-designed and useful for nouns, but look more artificial for other lexical categories. We show that it is the telic role of nouns which is the most useful. We also show how the internal structure of this role can be made more precise and its use more reliable and accurate by means of types and how it can be partitioned by means of types into ontological domains for modelling some forms of metaphors.

- Types are not sufficiently 'constrained' to account for the constraints holding, for each predicate, on the different sense/usage variations they may be subject to. We show that an underspecified Lexical Conceptual Structure (LCS) (Jackendoff 90) is more appropriate because of its ability to represent underspecified meaning and therefore the polymorphism of senses in the GL, because of the relevance and low-granularity of its primitives (that we have slightly enhanced).

- Elements of the Qualia structure can be incorporated into semantic composition rules to make explicit the semantics of the combination predicate-argument, instead of developing lexical redundancy rules.

- A rule-based approach (also used by other authors such as (Copestake and Briscoe 95), (Ostler and Atkins 92), (Numberg and Zaenen 79)) is contrasted with the Qualia-based approach to deal with sense shiftings and in particular selective binding, metaphors (that the GL cannot resolve a priori) and metonymies. Another view is presented in (Jackendoff 97) with the principle of enriched composition, which is in fact quite close to our view, but restricted to a few specific coercion situations (aspectual, mass-count, picture, begin-enjoy).

- The rules for type shifting we present here are not lexical rules, as in (Copestake and Briscoe $95)$, but they are part of the semantic composi- 
tion system, which makes them more general.

This paper is devoted to adjectival modification (see also (Bouillon 97, 98)). The goal is to study the use and impact of the Qualia structure of the modified noun in the determination of the semantic representation of the association Noun + Adjective.

To illustrate this study, we have chosen one of the most polysemic French adjectives: bon (good), which covers most of the main situations. Other adjectives, often cited in the GL literature, such as sad, fast, difficult or noisy have been studied and confirm this analysis. We observed also many similarities within semantic families of adjectives.

\section{Conceptual versus Lexicographic Analysis of Lexical Items}

In this section, we outline the differences but also the cooperation between conceptual and lexicographic analysis of the semantics of lexical items to build a lexicon suitable for the development of generative devices.

\subsection{Adjectives in technical texts}

We have considered a sample of technical texts in French from various origins and used a simple tagging and extraction system developed for our needs. We have considered a total of 386 pages of text, with a total of 193146 word occurences, among which, we have 14598 occurences of adjectives. These occurences correspond to 754 different adjectives, among which 720 are restrictive adjectives. We will only consider this latter set.

A small number of adjectives appear frequently:

\begin{tabular}{|c|c|}
\hline \multicolumn{2}{|c|}{ Fig. 1 Adjective frequencies } \\
\hline \hline interval & nb. of adjectives concerned \\
\hline$\geq 300$ & 5 \\
\hline$\geq 300$ and $\prec 150$ & 12 \\
\hline$\geq 150$ and $\prec 50$ & 81 \\
\hline \hline
\end{tabular}

This means that 98 adjectives appear relatively frequently in texts, i.e. only about $13.6 \%$ of the total. In terms of occurences, these adjectives cover 11887 occurences, i.e. about $81 \%$ of the occurences. Adjectives from eight main 'semantic' families appear frequently. These families do not correspond exactly to those defined by (Dixon 91) (see also an introduction in (Raskin et al. 95)), which look too vague (figures have been rounded up or down to the closest integer):

\begin{tabular}{|c|c|c|}
\hline \multicolumn{3}{|c|}{ Fig. 2 Adjective semantic families } \\
\hline \hline Name & example & freq. (\%) \\
\hline temporal & actuel, passé & 10 \\
\hline evaluative & bon, grand, cher & 24 \\
\hline locational & central, externe & 10 \\
\hline aspectual & courant, final & 8 \\
\hline technical & chimique & 17 \\
\hline nationalities & international & 3 \\
\hline shapes & rond, rectangulaire & 4 \\
\hline society, culture & economique, social & 6 \\
\hline others & - & 18 \\
\hline \hline
\end{tabular}

In terms of 'polysemic power', evaluative, locational, and shapes are the families which are the most polysemic, with a ratio of an average of 3.8 senses per adjective. Nationalities, technical and aspectual adjectives are much less polysemic.

\subsection{A conceptual analysis of adjectives}

The GL approach requires a conceptual analysis of adjectives in order to focus on a relatively small number of senses. The idea is to isolate generic conceptual 'behaviors', while taking also into account the constraints on linguistic realizations as in the lexicographic approach.

The principle that we attempt at validating is to define a 'deep' LCS representation for each predicative lexical item, which is generic enough to accomodate variations within a sense and precise enough to be meaningful and discriminatory w.r.t. other wordsenses. To be able to represent sense variations in an efficient and reliable way, the variable or underspecified elements should be 'low level' elements such as functions or paths. Semantic fields may also be altered, e.g. going from location to psychological or to epistemological (Pinker 93). Such an approach is being validated on various semantic families of verbs.

The variable elements seem to belong to various ontologies (a crucial topic under intense investigation), such as the ontology of events (active, sleeping, terminated, etc.), of people's quilities, etc.

\subsection{Meanings of bon}

In this short document, for the purpose of illustration, let us consider the adjective bon (corresponding quite well to good), which is one of the most polysemic adjective: 25 senses identified in WordNet (e.g. (Fellbaum 93)). In fact, bon can be combined with almost any noun in French, and as (Katz 66) pointed out, good would need as many different readings as there are functions for objects.

We have identified the following senses and sense variations (metaphors and metonymies in particular, expressed as in (Lakoff 80)):

1. Idea of a good working of a concrete object w.r.t. what it has been designed for: un bon tournevis, de bons yeux (good screw-driver, good 
eyes). Metaphors abound: e.g.: 'communication acts as tools': une bonne plaisanterie/mise au point (a good joke), 'function for tool' (un bon odorat), 'paths as tools' (a good road). 1 Metonymies are rather unusual since if $X$ is a part of $Y$, a good $X$ does not entail a good $Y^{2}$.

2. Positive evaluation of moral, psychological, physical or intellectual qualities in humans: bonne personne, bon musicien, (good person, good musician). The basic sense concerns professions and related activites or humans as a whole: it is the ability of someone to realize something for professions, and, for humans, the high level of their moral qualities (an enumeration can be given or a kind of higher-order, typed expression).

This second sense could be viewed as a large metaphor of the first, with a structurepreserving transposition to a different ontology: from tools to professional or moral skills.

There are some 'light' metaphors such as: 'social positions or ranks as professions' (a good boss / father / friend / citizen), and a large number of metonymies: 'image for person, image being a part of a person' (a good reputation), 'tool for profession' (a good scalpel), 'place for profession' (a good restaurant). These metaphors have a good degree of systematicity.

3. Intensifier of one or more properties of the noun, producing an idea of pleasure and satisfaction (this is different for sense 5$)^{3}$ :

noun(+edible): good meal/dish/taste = tasty, with metonymies such as 'container for containee' (a good bottle/ glass), noun(+fine-art): good film $/$ book/painting $=$ valuable. with metonymies such as 'physical support for contents' (good $C D$ ),

noun(+smelling): good odor, noun ( + psycho): good relation/ experience noun(+human relations): good neighbours.

Note that bon can only be used with neutral or positive nouns, we indeed do not have in French *good ennemies, *good humidity with the sense outlined here.

4. Quantification applied to measures or to quantities: a good meter, a good liter, a good

\footnotetext{
${ }^{1}$ In the combination noun + adjective, the noun is the element that undergo the metaphor. The adjective being a predicate, it is its relation to the noun it modifies which is metaphorical, similarly to the relation verb-noun. The semantics of the noun remains a priori unaltered.

${ }^{2}$ This needs refinements: there are some weak forms of upward inheritance in the part-of relation: e.g. if the body of a car is red, then the car is said to be red.

${ }^{3}$ Norms are being defined for about 600 top-most nodes of a general purpose ontology in different projects and research groups (e.g. NMSU, ISI, Eagles EEC project), they will be used as soon as available.
}

amount/salary, a good wind. In this case, good means a slightly more than the unit/measure indicated or above the average (for terms which are not measure units such as wind or salary). This sense being quite different since it is basically a quantifier, it won't be studied hereafter.

5. Idea of exactness, accuracy, correctness, validity, freshness, etc.: un bon raisonnement/calcul $=$ exact, accurate $(a$ good deduction/computation), good note/ticket $=$ valid, $a$ good meat $=$ fresh or eatable, a good use $=$ appropriate, good knowledge $=$ efficient, large and of good quality. The meaning of bon is therefore underdetermined. Depending on the noun, the semantics of bon is slightly different, this is not really a case of co-composition. It is the semantic type of the noun and that of the selected predicate in the telic role of the noun which determine the meaning of the adjective in this particular NP. We call this phenomenon, by comparison with selective binding, selective projection, because the meaning is projected from the noun's telic role. Sense 5 is substantially different from sense 1: it is basically boolean (e.g. exact or not), there is no idea of tool, function or even activity.

Bon appears in a large number of fixed or semi-fixed forms such as: le bon goût, le bon sens, le bon temps, une bonne giffle.

Almost the same behavior is observed for all evaluative adjectives such as excellent, terrific, bad or lousy in French. For example, for mauvais (bad), senses 1,2 and 3 are identical, sense 4 is only applicable to amounts (mauvais salaire), not to units and sense 5 is almost identical, it conveys the idea of erroneous deduction, invalid ticket, bad use and rotting meat. Note that in WordNet, bad has only 14 senses, whereas good has 25 senses, with no clear justification.

\subsection{A comparison with WordNet}

We have carried out a comparison of our conceptual analysis with the lexicographic analysis in WordNet. We have compared manually a subset of 54 adjectives among the above mentioned frequently used adjectives. Among these adjectives, 30 are polysemic in our approach while 44 belong to several synsets in WordNet:

\begin{tabular}{|c|c|c|}
\hline \multicolumn{3}{|c|}{ Fig. 3 A comparison with WordNet } \\
\hline \hline criterion & $(1)$ & $(2)$ \\
\hline total number of senses found & 114 & 256 \\
\hline average nb. of senses/item & 2.11 & 4.9 \\
\hline \hline
\end{tabular}

(1): Conceptual approach, (2) WordNet 1.6. 22 of our descriptions are close to WordNet (for adjectives which are not much polysemic) while 32 differ 
largely (for highly polysemic adjectives), for which our approach identifies much less senses.

\subsection{Underspecification versus polysemy}

Each of the senses of bon has many facets and interpretations depending on the noun it modifies. As for verbs or nouns (Busa 97), polymorphic types are used to represent the semantics of the expected nouns, viewed as arguments of the adjective predicate. The semantic representation associated with a sense is therefore underspecified and tuned to reflect this polymorphism. The scope of underspecified elements must however be bounded and precisely defined by 'lexical' types and by additional constraints. The generative expansion of underspecified fields can be defined from lexical items using a fix-point semantics approach (Saint-Dizier 96).

\subsection{Towards an automatic acquisition of conceptual descriptions}

Some on-line resources and dictionaries may efficiently contribute to this task. We have considered several mono- and bi-lingual dictionaries in order to evaluate convergences. Only those structured on a conceptual basis are worth considering. Among them, the Harrap's German-French dictionary is very nicely structured in a conceptual perspective, providing translations on an accurate semantic basis. Senses are slightly more expanded than in the GI approach to account for translation variations, but closely related senses can be grouped to form the senses defined above.

Another source of knowledge for English is Corelex ${ }^{4}$, which is just being made accessible. It contains word definitions specifically designed for the GL. Its evaluation is about to start.

\section{Generative Devices and Semantic Composition}

Let us now analyze from a GL point of view the meanings of the adjective bon.

In (Pustejovsky 95), to deal with the compound adjective+noun, a predicate in the telic of the noun is considered. For example, fast, modifying a noun such as typist, is represented as follows:

$\lambda e\left[\operatorname{type}^{\prime}(e, x) \wedge f a s t(e)\right]$

where e denotes an event. This formula says that the event of typing is fast. A similar representalion is given for long, in a long record. This approach is appropriate to represent temporal notions in a coarsegrained way, i.e. the event is said to be fast (with e.g. potential inferences on its expected duration) or long. But this approach is not viable for bon, and many other adjectives with little or no temporal dimension. In:

\footnotetext{
4 available at:

www.cs.brandeis.edu/paulb/CoreLex/corelex.html
}

$\lambda e\left[\operatorname{type}^{\prime}(e, x) \wedge \operatorname{good}(e)\right]$

it is not the typing event which is 'good' but the way the typing has been performed (certainly fast, but also with no typos, good layout, etc.). A precise event should not be considered in isolation, but the representation should express that, in general, someone types well, allowing exceptions (some average or bad typing events). This involves a quantification, more or less explicit, over typing events of $x$. Finally, bon being polysemous, a single representation is not sufficient to accomodate all the senses.

As introduced in section 1, the semantic representation framework we consider here is the LCS. The nature of its primitives and its low-level granularity seem to be appropriate for our current purpose. Underdetermined structures are represented by a typed $\lambda$-calculus.

\section{1 sense 1: Bon = that works well}

This first sense applies to any noun of type tool, machine or technique: a good car, a good screwdriver. The semantic representation of bon requires a predicate from the telic role of the Qualia structure of the noun. It is the set (potentially infinite) of those predicates that characterizes the polymorphism. We have here a typical situation of selective binding (Pustejovsky 91), where the representation of the adjective is a priori largely underspecified. Let us assume that any noun which can be modified by bon has a telic role in which the main function(s) of the object is described (e.g. execute programmes for a computer, run for a car ${ }^{5}$ ), then the semantics of the compound adjective + noun can be defined as follows:

Let $\mathrm{N}$ be a noun of semantic type $\alpha$, and of Qualia: [..., Telic: T, ...]

where $T$ denotes the set of predicates associated with the telic role of the noun $\mathrm{N}$. Let $\mathrm{Y}$ the variable associated with $\mathrm{N}$ and let us assume that $\mathrm{T}$ is a list of predicates of the form $F_{i}(.,-)$. Then the LCS-based representation of bon is:

$\lambda Y: \alpha, \lambda F_{i},\left[\right.$ state $B E_{+c h a r, t i d e n t}([$ thing $Y]$, [+prop $\left.\left.\left.A B I L I T Y-T O\left(F_{i}(Y,-)\right)=h i g h\right]\right)\right]$.

which means that the entity denoted by the noun works well, expressed by the evaluation function ABILITY-TO and the value 'high'. This type of low-level function abounds in the LCS, this principle is introduced in (Jackendoff 97). Note that the second argument of the predicate $F_{i}$ does not need to be explicit (we use the Prolog notation '-' for these positions).

The Qualia allows us to introduce in a direct way a pragmatic or interpretative dimension via the instanciation of $F_{i}(-,-)$.

\footnotetext{
${ }^{5}$ Less prototypical predicates can also be considered, e.g. comfort or security for a car, which are properties probably described in the constitutive role of the Qualia of car.
} 
The constant 'high' can be replaced by a more accurate representation, e.g. 'above average', but the problem of evaluating a functionality remains open. More generally, the introduction of low level functions, such as ABILITY-TO, and specific values, such as 'low', should be introduced in a principled way, following the definition of ontologies of different domains, e.g. action, intensities, etc. This is quite challenging, but necessary for any accurate semantic framework.

Note finally that instead of quantifying over events, bon is described as a state: the functionalities of the object remain good, even when it is not used effectively. If several functionalities are at stake, we may have a conjunction or a more complex combination of functions $F_{i}$.

From a compositional point of view, the combination Adjective + Noun is treated as follows, where $R$ is the semantic representation of the adjective, $T$, the contents of the telic role of the Qualia of the noun $\mathrm{N}$ of type $\alpha, \tau$, a particular element of ' $\mathrm{T}$, and $Y$, the variable associated with the noun: sem-composition $(\operatorname{Adj}(\mathrm{R}), \operatorname{Noun}($ Qualia $(\mathrm{T}))=$ $\lambda Y: \alpha, \exists F_{i}\left(Y_{-}\right) \in T$, $\left(N(Y) \wedge R(Y)\left(F_{i}\left(Y_{,-}\right)\right)\right)$.

The open position in $\mathrm{R}(\mathrm{Y})$ is instanciated by $\beta$ reduction. The selection of $F_{i}$ is simple: for basic tools, there is probably only one predicate in the Qualia (screw-driver $\rightarrow$ screw), for more complex nouns, there is an ambiguity which is reflected by the non-deterministic choice of $F_{i}$, but probably organized with preferences, which should be added in the Qualia. It is the constraint on the type of $Y$ that restricts the application of that semantic composition rule. This notation is particularly simple and convenient.

Metaphors are treated in a direct way: the constraint on the type of $Y$ can be enlarged to:

$\lambda Y: \beta \wedge \alpha$, metaphor $(\beta, \alpha)$

and the remainder of the semantic composition rule and semantic formula remains unchanged. We have, for example:

metaphor(communication - act,tool) (joke). metaphor(communication - path, tool) (road).

which is paraphrased as 'communication path viewed as a tool'.

We have evaluated that, in French, there are about 12 frequent forms of metaphors for this sense. The study of this first sense suggests that the introduction of a hierarchy of preferences would be a useful extension to the Telic role, reflecting forms of prototypicality among predicates.

\subsection{Sense 2: Bon restricted to cognitive or moral qualities}

Another sense of bon modifies nouns of type profession or human. The treatment is the same as in the above section, but the selection of the predicate(s) $\tau=F_{i}(X, Y)$ in the telic of the noun's qualia must be restricted to properties related to the moral behavior (makes-charity, has-compassion, has-integrity) when the noun is a person; and to some psychological attitudes and cognitive capabilities when the noun denotes a profession (e.g. $a$ good composer). Alternatively, some of these properties could be found in the constitutive role (approximately the part-of relation), if properties can be parts of entities.

The typing of the predicates in the Qualia roles can be done in two ways, (1) by means of labels identifying the different facets of a role, as in (Bergler 91) for report verbs, but these facets are often quite ad'hoc and hard to define, or (2) by means of types directly associated with each predicate. These types can, for example, directly reflect different verb semantic classes as those defined in (Levin 93) or (Saint-Dizier 96) on a syntactic basis, or the major ontological classes of WordNet or EuroWordNet and their respective subdivisions. This solution is preferable, since it does not involve any additional development of the Telic role, but simply the adjunction of types from a separate, pre-defined ontology. The WordNet or EuroWordNet types also seem to be quite easy to handle and well-adapted to the phenomena we model. This remains to be validated on a large scale.

An LCS representation for this sense of bon is, assuming the following types for $F_{i}$ :

$\lambda Y$ : human, $F_{t}$ : action - related - to profession $\vee$ moral-behavior, $Y: \alpha$,

[state $B E_{+ \text {char, }+ \text { ident }}([$ theng $Y]$,

$\left[+\right.$ prop $\left.\left.\left.A B I L I T Y-T O\left(F_{i}(Y,-)\right)=h i g h\right]\right)\right]$.

When several predicates are at stake, a set of $F_{i}(Y,-)$ can be considered in the representation, or the statement is ambiguous.

Metonymies such as a good scalpel are resolved by the general rule: 'tools for professions'. This information could be in a knowledge base or, alternatively, it can be infered from the Telic role of the tool: any instrument has a predicate in its telic role that describes its use: the type of the first argument of the predicate is directly related to the profession that uses it. For example, scalpel has in its telic role: cut $(X:$ surgeon $\vee$ biologist, $Y:$ body).

When the profession is identified, the standard procedure for determining the meaning of the compound can be applied. Metonymies using the part-of relation are quite simple to resolve using the constitutive role, as in the GL.

\subsection{Sense 3: Bon as an intensifier}

Another main role of bon is to emphasize a quality of the object denoted by the noun. As shown in section 2 , there is a certain action associated with the telic of the modified noun that produces a certain pleasure. 
For example, watching a good film entails a certain pleasure.

Let us consider again a noun $\mathrm{N}$ of type $\alpha$ (e.g. edible object) associated with the variable $Y$. The entity (human) undergoing the pleasure is not explicit in the NP, it is represented by $X$, and included in the scope of a $\lambda$-abstraction. Let $F_{i}(X, Y)$ be the predicate selected in the telic role of $N$. The LCS representation is then:

$$
\begin{aligned}
& \lambda X: \text { human, } Y: a, F_{i}(X, Y) \\
& \text { [event } C A U S E\left(\left[\text { event } F_{i}(X, Y)\right],\right. \\
& {\left[\text { state } B E_{+p s y}([\text { thtng } X],\right.} \\
& \left.\left.\left.\left.\quad\left[\text { place } A T_{+p s y}([+ \text { place pleasure }])\right]\right)\right]\right)\right] .
\end{aligned}
$$

We have here another form of representation for bon, where $F_{i}$ is a CAUSE.

The term 'pleasure' is an element of an ontology describing e.g. mental attitudes and feelings. It is relatively generic and can be replaced by a more precise term, via selective projection (see below for sense 5 ), depending on the nature of the pleasure.

An alternative representation describes a path towards the value 'pleasure', giving an idea of progression:

$\lambda X:$ human, $Y: \alpha, F_{i}(X, Y)$

[event CAUSE $\left(\left[\right.\right.$ event $\left.F_{i}(X, Y)\right]$,

[event $G O_{+p s y}([$ thing $X]$,

[path TOWARDS $S_{+p s y}([$ +place pleasure $\left.\left.\left.\left.\left.])\right]\right)\right]\right)\right]$.

Notice that this sense of bon does not imply an idea of quantity: a good meal does not entail that the meal is big, a good temperature does not entail that the temperature is high, but rather mild. The semantic composition rule is similar as in 3.1 .

The metonymy 'container for containee' (a good bottle) is resolved by a type shifting on $\mathrm{Y}$. $\mathrm{Y}$ may be of type $\beta$ iff:

$\exists Z: \alpha, Y:$ container $\wedge$ container - for $(Y, Z)$. Inferences are identical for e.g. a good $C D$.

\subsection{Sense 5: Bon = exact or correct}

We have here a situation of selective projection: the exact meaning of bon is projected from the type of the modified noun and the type of the predicate selected in the noun's Telic role.

For example, if the noun is of type bank - note $V$ ticket and the type of the predicate selected in the noun's Telic role is pay $\vee$ give - access - to, then the meaning of bon is 'valid':

$\lambda X:$ bank - note $\vee$ ticket,

[state $B E_{\text {+char, }}$ tident $\left(\left[\right.\right.$ thing $\left._{\text {thin }} X\right]$,

[place $A T_{\text {+char, }}$ ident $([+$ propvalid $\left.\left.\left.(X)])\right]\right)\right]$.

The constraint on the type of the telic role is stated in the semantic composition rule:

sem-Composition $(\operatorname{Adj}(\mathrm{R}), \operatorname{Noun}(\mathrm{X}, \mathrm{Qualia}(\mathrm{T})))=$

$\lambda X:$ bank - note $\vee$ ticket,

$\exists F_{i}(-,-):$ pay $\vee$ give - access - to $\in T$,

$(N(X) \wedge R(X))$.

It is necessary to have both a constraint on the noun and on the predicate(s) in the telic role: (1) the type of the predicate in the telic role is certainly not a sufficient constraint, e.g. every noun's telic role in which there is the predicate pay cannot be combined with bon with sense 5 ; (2) the constraint on the type of the noun is also not sufficient, e.g. a medecine is a kind of food, but we don't eat it.

\section{Representing the core meaning of a word-sense}

The work presented here has shown the necessity of describing the semantics of a lexical item at a relatively 'deep' level, in order to make explicit the meaning elements subject to alterations in the sense. variations shown above. It turns out, so far, that these elements can be represented by LCS primitives and a few functions and values, assumed to belong to general-purpose, and often commonly-admitted, ontologies. This remains an assumption since this type of ontological knowledge is still under development, but the elements used are relatively simple and standard. Besides ontologies, and not very far from them, we also find information contained in the noun's Qualias, but in a less structured way, making selection more difficult.

Core meaning definition requires a good analysis of a word-sense and of its behavior in different contexts. This is however not so difficult to elaborate once the formalism is stabilized. Also, we noted that semantically close words share a lot, making descriptions easier. This is in particular true for verbs. Besides adjectives, we have also studied a number of different types of verbs, as e.g. the verb couper (cut), often used as an example in the literature. Its core representation would be the following:

$\lambda I, J$ [event CAUSE([thıng I],

[event $G O_{A}(X,[$ path $\left.\left.\left.Y])\right]\right)\right]$.

with the following values for the core sense:

$\mathrm{A}=+$ loc $; \mathrm{X}=$ [thing PART $-O T(J)]$

$\mathrm{Y}=A W A Y-F R O M_{A}($ [place $\left.L O C A T I O N-O F(J)]\right)$

For the metaphor: 'to cut a conversation/ a film, etc...', the values for the above variables become:

$\mathrm{A}=+$ char, tident, $\mathrm{X}=[$ event/state $J]$

$\mathrm{Y}=A W A Y-F R O M_{A}([$ prop $A C T I V E(J)])$

where $\operatorname{ACTIVE}(\mathrm{J})$ is an elementary property of an ontology describing the status of events. A conversation is viewed as a flow which becomes non-active. A similar treatment is observed for other types of metaphors, with elliptic forms, such as couper l'eau/ l'électricité/ les crédits, also viewed as flows. The property AVAILABLE(J) will then be used, which is at a comparable abstract level in an ontology than ACTIVE(J).

\section{Long-distance compositionality}

The NP a good meat is related to senses 2 or 5 , it therefore includes in its domain of meanings structures presented in sections 3.2 and 3.4. Instead of 
choosing one solution solution (a generate and test strategy), a set can be provided (as in constraint programming). Now, if we have an NP of the form: une viande bonne à consommer, then the parsing of consommer will provoque the selection of sense 5 (and subsense 'fresh/consumable' via selective projection) because of the type of consommer. If, conversely, we have une viande bonne à déguster, then, since déguster is of type 'eat.enjoy' (a dotted type in the GL), sense 2 is selected. The space of meanings is restricted when additional information is found.

A second case involves default reasoning (as in (Pernelle 98)). In un bon couteau pour sculpter (a good knife to carve), by default, the action that the knife performs well is that protypically found in its telic role. But, if a less prototypical action is found explicitly in the sentence, then this latter is prefered and incorporated into the semantic representation instead of the default case. Indeed, the telic role describes prototypical actions, since the others are often unpredictable. The default meaning of bon is kept and 'frozen' until the whole sentence has been parsed. If there is no contracdiction with that sense, then it is assigned to the adjective, otherwise, it is discarded in favor of the sense explicitly found in the sentence.

Finally, we consider the expressions $Y$ makes a good $X, Y$ is a good $X$ as collocations where good is not fully treated compositionally.

\section{Conclusion}

In this paper, we have presented an analysis of adjectival modification within the GL perspective, with the illustration of the French adjective bon. We have proposed several extensions to the Telic role to be able to account for the representation of the different forms of sense variations. In particular, we have shown how types can be added, and how predicates from the telic participate to the construction of the semantic representation of the compound noun + adjective.

Coercions and the treatment of metaphors and metonymies are generally assumed to be general principles, however, they are in fact more specialized than they seem at first glance (e.g. une bonne toque/ plume $=$ a good cook/ writer is quite specific, or very constrained). It is then necessary to introduce narrow selectional restrictions on their use. Also, the similarities, quite important, outlined between the different cases presented here and observed for other families of adjectives suggest that there is a common typology for adjectival modification. What then would be a general formalism? How much are these rules subject to linguistic variation?

Acknowledgements I thank James Pustejovsky, Federica Busa and Françoise Gayral for discussions which helped improving this work.
I don't thank my university administration, in particular the accounting dept., who made every possible effort to make this research more difficult.

\section{References}

Bergler, S., (1991) The semantics of collocational patterns for reporting verbs, in proc. 5th EACL.

Bouillon, P., Mental State Adjectives: the Perspective of Generative Lexicon, in proc. Coling'96, Copenhaguen, 1996.

Bouillon P., Polymorphie et sémantique lexicale, Thèse de troisième cycle, Université de Paris VII, 1997.

Busa, F., (1996), Compositionality and the Semantics of Nominals, PhD. Dissertation, Brandeis University, MA.

Copestake, A., Briscoe, T., (1995), Semi-Productive polysemy and sense extension, journal of semantics, vol. 12-1.

Dixon, R.M. W., (1991) A new approach to English grammar on semantic principles, Oxford, Clarendon Press.

Fellbaum, C., (1993), "English Verbs as Semantic Net", Journal of Lexicography.

Jackendoff, R., (1990), Semantic Structures, MIT Press.

Jackendoff, R., (1997), The Architecture of the Language Faculty, MIT Press.

Katz, G. (1966), The philosophy of Language, Harper and Row, New-York.

Lakoff, G., Johnson, M. (1980), Metaphors we Live $B y$, University of Chicago Press.

Levin, B., (1993), English verb Classes and Alternations: A Preliminary Investigation, Chicago Univ. Press.

Nunberg, G.D., Zaenen, A., (1992), Systematic Polysemy in Lexicology and Lexicography, proc Euralex92, Tampere, Finland.

Ostler, N., Atkins, S., (1992), Predictable Meaning Shifts: some lexical properties of lexical implication rules, in J. Pustejovsky and S. Bergler (eds.) Lexical Semantics and Knowledge Representation, Springer Verlag.

Pernelle, N., (1998), Raisonnement par défaut et lexique génératif, $\mathrm{PhD}$ dissertation, LIPN, Paris.

Pinker, S., (1993), Learnability and Cognition, MIT Press.

Pustejovsky, J., (1991), The Generative Lexicon, Computational Linguistics, vol 17-4.

Pustejovsky, J., (1995), The Generative Lexicon, MIT Press.

Raskin, V., Niremburg, S., (1995) Lexical semantics of adjectrives, a micro-theory of adjectival meaning, MCCS report 95-288.

Saint-Dizier, P. (1986) A Logic Programming interpretation of Type Coercion inthe generative lexicon, in proc. NLULP'96, Lisbon.

Saint-Dizier, P., (1996), Verb semantic classes based on 'alternations' and on WordNet-like semantic criteria: a powerful convergence, in proc. Predicative Forms in Natural language and in lexical knowledge bases, IRIT, Toulouse. 